\title{
Uma ontologia para a cadeia produtiva de sementes
}

\section{Selma Josette Silveira Debtil ${ }^{1}$, Luma Alves Lopes ${ }^{1}$, Hugo Leonardo Petla ${ }^{1}$ and Maria Salete Marcon Gomes Vaz ${ }^{1}$}

\author{
${ }^{1}$ Programa de Pós-Graduação em Computação Aplicada - Universidade Estadual de Ponta Grossa \\ *sdebtil@yahoo.com.br; luma_lopes@outlook.com; hugopetla@hotmail.com; salete@uepg.br
}

Recebido: 08/12/2018. Revisado: 15/05/2019. Aceito: 03/06/2019.

\section{Resumo}

A utilização de processos de rastreabilidade proporciona segurança e qualidade aos produtos. Esses fatores são importantes para a produção de sementes, cuja a preocupação pela utilização de sementes certificadas e fiscalizadas vem crescendo. O objetivo desse artigo é apresentar o desenvolvimento de uma ontologia, como forma de representar o conhecimento, para garantir a qualidade e melhoria dos processos da rastreabilidade da cadeia produtiva de sementes. Para a construção da antologia, realizou-se o levantamento de leis, regulamentos e normativas inerentes a produção de sementes. Foi utilizada a ferramenta Protégé com a combinação das metodologias Enteprise, Methontology, On-toKnowledge e do guia Ontology Development 101. Como resultado, foi obtido um sistema de representação de conhecimento com sessenta e nove classes. $O$ roteiro metodológico dessa pesquisa, possibilita a obtenção dos principais termos do domínio e a taxionomia gerada na ontologia, o que possibilita a sua utilização por outros sistemas de rastreabilidade de sementes.

Palavras-Chave: Processo de rastreabilidade; Representação de conhecimento; Sementes; Vocabulário

\section{Abstract}

The use of traceability processes provides product safety and quality. These factors are important for the production of seeds, whose concern for the use of certified and supervised seeds has been increasing. The objective of this article is to present the development of an ontology, as a way to represent knowledge, to guarantee the quality and improvement of the traceability processes of the seed production chain. For the construction of the anthology, it was carried out the survey of laws, regulations and norms on the production of seeds. The Protégé tool and the Enteprise, Methontology, On-toKnowledge and Ontology Development 101 methodologies were used. As a result, a knowledge representation system with sixty-nine classes was obtained. The methodological script of this research, allows to obtain the main terms of the domain and the taxionomia generated in the ontology, which allows its use by other systems of traceability of seeds.

Key words: Knowledge representation; Seeds; Traceability process; Vocabulary

\section{Introdução}

As ontologias representam o conhecimento e estão atreladas à evolução tecnológica. A sua aplicação é crescente em setores de gestão do conhecimento, na mineração de dados e está intrinsecamente ligada à evolução da Web. A agricultura, por sua vez, demanda constantemente de controles atualizados e em conformidade com a legislação de cada cultura.

Na agricultura, para que a produção de alimentos tenha sucesso, são utilizados diversos recursos tecnológicos. Uma das formas de melhorar a produtividade é a utilização de sementes de qualidade. A semente possui atributos de qualidade genética, física, fisiológica e sanitária, que proporcionam garantia de um bom desempenho agronômico.

O controle pela utilização de sementes de qualidade e de alto vigor, gera a necessidade de utilização de processos de rastreabilidade. A ausência de 
um programa de rastreabilidade impede a devida responsabilização e a tomada de ações preditivas, preventivas e corretivas.

No Brasil, por exemplo, o MAPA - Ministério da Agricultura, Pecuária e Abastecimento, por meio da Lei $\mathrm{n}^{0}$ 10.711, de 05 de agosto de 2003, descreve uma série de exigências que devem ser seguidas na produção de sementes. Quem deseja realizar qualquer atividade relacionada com sementes, deve seguir as determinações da legislação e procurar assessoria nas Superintendências Federais de Agricultura (SFA'S), gerando para os produtores uma série de procedimentos a serem adotados para garantir a qualidade em todas as etapas do seu ciclo, incluindo a origem genética e acompanhamento de gerações.

Todo o processo de rastreabilidade gera um grande número de informações. Essas informações precisam estar disponíveis, ser transparentes, acessíveis, com acesso amplo, reutilizáveis e efetivamente aproveitadas. Porém, as informações acarretam novos desafios em suas estruturações como, por exemplo, a de que os dados possam ser integrados de forma consistente.

Com a ontologia é possível capturar o conhecimento de um domínio, transformá-lo em conceitos e relacionamentos, para que seja possível descrever um vocabulário formal, a fim de compartilhar e reutilizar a informação (Andrade; 2008). O desenvolvimento de uma ontologia é similar à definição e estruturação de conjunto de dados para que sejam utilizados.

A criação de um sistema de rastreabilidade a partir de uma ontologia, torna o processo de criação mais rápida ao possibilitar que sejam compartilhados e reutilizadas informações sobre o domínio.

Desta forma, o objetivo deste artigo é apresentar uma ontologia para rastreabilidade na cadeia produtiva de sementes. O desenvolvimento dessa ontologia contribuirá para todas as fases do processo de produção, integração dos agentes envolvidos no processo e as informações aplicáveis e disponíveis ao consumidor final.

\section{Ontologia e Rastreabilidade}

Ontologia na Ciência da Computação trabalha o significado e a existência de objetos e conceitos que existem no domínio de um sistema, seus relacionamentos e os seus significados.

Ontologia foi adotada pelas comunidades de inteligência artificial e gestão de conhecimento, para se referir aos conceitos e termos, usados para descrever alguma área do conhecimento ou construir uma representação, com a descrição formal de conceitos e relacionamentos (Guariano; 1998). Uma ontologia de determinada área de conhecimento provê semântica, é processável por máquina, com bases de conhecimento interoperáveis e melhor estruturadas.

O estudo de ontologias caracteriza-se como um ramo de pesquisa, propondo uma nova maneira de representar o conhecimento.

Para representar os conceitos, as relações entre os conceitos e a semântica de um domínio de conhecimento, é estudada uma série de formalismos, gerando um modelo formal com declarações lógicas representando o conhecimento do domínio, a ser manipulado em um sistema computacional.

No entanto, não há uma definição universal para ontologias. Uma razão dessas inúmeras definições é a grande quantidade de formas de suas aplicações. $\mathrm{Na}$ área agrícola como na computação, cresce o uso de ontologias no processo de representação de informação para extrair conhecimento.

Já a rastreabilidade, refere-se à habilidade de descrever e seguir o ciclo de vida de um elemento. Esse rastreamento pode acontecer a partir da origem, ou de qualquer ponto do desenvolvimento até a utilização dos produtos, ou então do caminho inverso, da sua utilização até a sua origem (Mohan and Ramesh; 2002).

Inserir informações detalhadas sobre a origem e as características dos produtos, distribuídos de acordo com lotes homogêneos, nas várias etapas da cadeia produtiva, tornou-se um instrumento de vantagem comercial. A possibilidade de obter informações mais detalhadas ou mesmo comprovar a autenticidade da origem da semente, consultando uma base de dados pelo código do produto é um diferencial do produtor.

Por meio do acesso aos registros, das informações e manuseio dos dados em qualquer ponto da cadeia produtiva, a rastreabilidade garante a qualidade dos produtos e proteção aos consumidores, ao possibilitar a eliminação de produtos alimentícios defeituosos e promover a investigação das causas dos problemas (Badia-Melis et al.; 2015).

Um sistema de rastreamento eficiente deve ser composto de normas e/ou referências da qualidade para garantir e preservar (GS1; 2012):

- Procedimentos estabelecidos;

- Relação de insumos permitidos e proibidos;

- Períodos de carência ou transição baseados em normas;

- Exigências dos produtores para que mantenham comprovantes de compras e de vendas; e

- Auditorias e vistorias surpresas e periódicas.

A ausência de um programa de rastreabilidade impede a devida responsabilização e a tomada de ações preditivas, preventivas e corretivas, já que a rastreabilidade é uma ferramenta utilizada para a identificação da origem do problema.

\section{Material e Métodos}

A aquisição do conhecimento foi embasada pela revisão bibliográfica conforme segue:

- Levantamento das Leis, Regulamentos e Normativas relacionadas à Rastreabilidade de sementes no Brasil, suas exigências e certificações; - (ii) Análise de sistemas de gestão da qualidade como HACCP - Hazard Analysis and Critical Control Point (HACCP; 2009) , ISO - International Organization for Standardization (ISO:22005; 2007) and GTS - Global Traceability Standard (GS1; 2012);

- Estudo dos sistemas de rastreabilidade de sementes existentes, para servir de parâmetro no desenvolvimento da Ontologia; e

- Análise do fluxo do processo de produção de sementes. 
Utilizou-se o Caderno de Campo que é um conjunto de documentos para registrar, regularmente, informações sobre processos e práticas de cultivo conduzido em áreas sob o regime de Produção Integrada. Os formulários com requisitos, obrigações e compromissos devem ser preenchidos durante o processo produtivo da semente. Toda atividade desenvolvida deve ser registrada, mesmo que não esteja contemplada neste caderno (Fernandes and Tibola; 2009).

As informações do Caderno de Campo englobam dados sobre: o produtor/empresa, responsável técnico, informações da parcela, dados climáticos, relação de máquinas, revisão de máquinas, tratos culturais, irrigação, adubação - macronutrientes, adubação - micronutrientes, adubação orgânica, fertirrigação - macronutientes, monitoramento de doenças, monitoramento de pragas e dados sobre a colheita.

Para a construção da ontologia, utilizou-se as metodologias Enterprise (Dietz; 2006), Methontology (Corcho et al.; 2005), On-to-Knowledge (Sure et al.; 2004) e do guia Ontology Development 101 (Noy and Mcguinness; 2001). Na especificação dos requisitos da ontologia, a On-to-Knowledge contribui por meio do emprego de questões de competência como modo simples e direto para confirmar o propósito e o escopo de uma ontologia.

Na documentação e na avaliação de ontologias há contribuição da Enterprise e a Methontology. Uma visão clara de como se dá um processo iterativo para o desenvolvimento de ontologias é demonstrado pela Ontology Development 101.

A ontologia para rastreabilidade na cadeia produtiva de sementes foi elaborada a partir da Web Ontology Language (OWL) (W3C; 2018), utilizando conceitos de classe e propriedade. As fases "Definir classes e hierarquia", "Definir propriedades das classes", "Definir as restrições das propriedades", "criar instâncias das classes", que compõem a metodologia utilizada, serão apresentadas em conjunto.

A ferramenta Protégé (C apresenta vários recursos para as atividades de implementação e avaliação de ontologias (Musen; 2015). Dessa forma, para o desenvolvimento da ontologia foi utilizada a ferramenta Protégé(C) 4.1.0 (OWL 2.0 (C)), que oferece uma interface gráfica e um ambiente interativo para construção de ontologias. É uma ferramenta de código aberto, mundialmente conhecida, provendo um conjunto de funcionalidades para construir modelos de domínio e aplicações baseadas em conhecimento com ontologias. A compilação de todos os conhecimentos capturados é realizada com a linguagem OWL (C) a partir da interface disponibilizada pela ferramenta Protégé( .

Optou-se pela utilização da OWL DL por estar incorporada ao Protégé(C) 4.1.0, com suporte a OWL 2.0 (C), em que uma das linguagens é a OWL2 DL (C).

Por fim, utilizou-se Reasoner que são utilizados para inferir informações que não são explicitamente representadas na ontologia (Semeráth et al.; 2017). Os reasoning padrões são: Subsumption checking, Equivalence checking, Consistency checking e Instantiation checking. A inicialização do Reasoner foi realizada por meio do Algoritmo Pellet.
A atualização da Ontologia e a validação a qualquer tempo é realizada por meio da utilização da ferramenta Methontology.

\section{Trabalhos correlatos}

Silva (2018) propôs uma ontologia para a rastreabilidade de dados agrícolas, mapeando o processo de produção da erva-mate. Nesse trabalho, utilizou a ferramenta Protégé (C e aplicou a Metodologia Ontology Development 101. Como resultado, obteve-se um sistema para melhoria do intercâmbio de dados para a cultura da erva-mate.

Em Abrahão and Hirakawa (2017) é proposto uma ontologia de tarefas para a representação do conhecimento técnico das operações do campo agrícola. A análise envolveu determinar os agentes e suas funções, os recursos de entrada, a decomposição de tarefas e subtarefas, controle dos fluxos, o conceito de tarefas, atributos e relações. Para verificar a consistência do modelo, foi realizado um estudo de caso na operação da colheita da cana-de-açúcar.

Melo (2017) propôs uma ontologia para o ciclo de exploração agrícola, com o objetivo de facilitar a interpretação e tradução de dados entre modelos de informação utilizados pelos produtores e organizações. Para a construção da ontologia, utilizou os modelos de informação do Zoneamento Agrícola de Risco Climático e do Banco Central do Brasil, além de planilhas de centro de custo. Como forma de validar o modelo, realizou a coleta de informações sobre a produção de café.

Em Macedo and Dias (2016) é apresentado o OntoTox, uma ontologia para doenças causadas pelo uso indevido de agrotóxicos. A representação do conhecimento envolve dados sobre os agrotóxicos, doençãs e intoxicações que podem ser causadas pelo uso indevido desses produtos. O OntoTox foi constrituído utilizando o Methontology, bem como algumas fases do método 101 e implementado utilizando a ferramenta Protégé (C)

"Rastreabilidade Online", o sistema de rastreamento foi desenvolvido por Gazzola-Neto et al. (2012), juntamente com a empresa Checkplant e a Sementes Oilema com a finalidade de controle da qualidade, informações técnicas e o histórico de produção de sementes de soja, em plataforma web, para tornar o acesso à informação rápido e seguro. O sistema consta de três módulos. O primeiro tem a função de receber e registrar informações da empresa produtora de sementes, suas fazendas de produção ou cooperados. O segundo, responsável pela comunicação do sistema online com as impressoras. O terceiro destinado a consulta dos códigos de rastreabilidade. A identificação ocorreu com a utilização de QR-Code, gravado com as informações sobre o histórico da produção, como, localização geográfica da fazenda com fotos, informações técnicas sobre o tratamento das sementes e resultados das avaliações do controle de qualidade.

Na Simulação de base ontológica em fluxo de água em solos orgânicos, aplicada a cana-de-açúcar na Flórida, de Kwon et al. (2010), foi apresentado o simulador OntoSim, como um ambiente de modelagem de dados única. Os processos solo-planta- 
nutrientes são representados como objetos de banco de dados e as relações definidas pelo usuário entre os objetos, e são usadas para gerar código de computador (em Java) para a execução da simulação. O objetivo foi modelar processos hidrológicos de cana cultivadas em solos orgânicos.

Este modelo OntoSIM-Sugarcane descreve a complexa hidrologia de subirrigação e drenagem em vala aberta, comumente usada em fazendas. $O$ OntoSim, foi originalmente, desenvolvido para ajudar os produtores de cítricos a otimizar os rendimentos de uma forma economicamente eficaz (Beck et al.; 2010). No entanto, OntoSim pode fornecer uma plataforma para desenvolver qualquer modelo de simulação conceitual e matemático.

A ênfase principal foi feita na modelagem de fluxo de drenagem vertical e lateral, na zona saturada, para facilitar os sistemas de simulação de controle de água, comumente utilizados em fazendas de cana-deaçúcar, com sub irrigação e drenagem em vala aberta. A calibração e validação destes processos foram realizadas, utilizando dados agrícolas recolhidos durante um período de quatro anos, seguido por uma discussão sobre futuras aplicações do OntoSim a outros ambientes de modelagem.

Papajorgji and Pardalos (2009) cobre a modelagem de sistemas com várias abordagens de modelos, inclusive, pela discussão do projeto e uso de ontologias. Fornece uma descrição clara sobre ontologias e dá informações sobre como a UML (Unified Modeling Language) pode ser usada para modelar ontologias em sistemas agrícolas, apresentando um projeto de Gestão de Informações de Fazenda, onde é ilustrado o uso de UML no projeto de ontologias e são comparadas UML e OWL. As aplicações de simulação de base ontológicas, na área de solo, água, e a gestão dos nutrientes utilizam o sistema de gerenciamento de ontologias LYRA, baseado em OWL. Este primeiro passo pode abrir diferentes oportunidades tais como em elementos de compartilhamento, entre os modelos que têm subsistemas semelhantes, ou envolvendo bancos de dados que podem ser integrados em modelos que utilizam esta abordagem.

Blaudete and Vilela (2007) relatam a importância das Unidades Beneficiadoras de Sementes para o processo de qualidade das sementes, que se consolida após seu beneficiamento, ou seja, todas as etapas pelas quais as sementes passam até ficarem prontas para semeadura. As Unidades beneficiadoras necessitam de informações preliminares para o seu planejamento, tais como avaliação do local (meios de transporte, mão-de-obra, condições climáticas), condições de beneficiamento (espécies, cultivares, épocas de semeadura e colheita) e condições de armazenamento (tipo e período de armazenamento). Por ser considerada a vitrine da empresa produtora de sementes, começa a ganhar importância, e seu planejamento deve seguir normas e procedimentos adequados que permitam adquirir sementes com a qualidade desejada.

Os principais processos em que as sementes de sojas foram submetidas dentro das unidades envolve a Recepção, Moega, Pré-Limpeza, Secador, Silo Armazenador, MAP, Espirais, Padronizador, Mesa de Gravidade, Tratadora e Balança Ensacadora (Blaudete and Vilela; 2007).
Leonelli (2006) desenvolveu o Modelo RIG Rastreabilidade Integrada de Grãos, com a finalidade de propor passos gerenciais que possibilitem promover a gestão da rastreabilidade nas diferentes etapas da cadeia produtiva. Foi sugerido o controle de três etapas: i) Condicionantes à adoção de mecanismos de identificação e rastreabilidade; ii) Planejamento das Atividades; e iii) Gestão das Operações.

Tillmann (2006) apresenta uma metodologia específica para implantação de um modelo de sistema integrado de gestão da qualidade adequado às unidades de beneficiamento sementes. A pesquisa orienta para gestão dos principais processos envolvidos no beneficiamento de sementes, fornece subsídios para facilitar a implantação de um sistema integrado de gestão da qualidade nas unidades beneficiadoras e, elabora um modelo de gestão da qualidade aplicado a todos os processos gerados nas unidades. A pesquisa ressalta os benefícios advindos com a implantação do modelo com a melhora na gestão dos processos, melhoria no desempenho organizacional e a satisfação dos clientes.

Além desses trabalhos, outras pesquisas também serviram de base para a especificação da Ontologia de Rastreabilidade para sementes como, por exemplo, os trabalhos de Zanatta and Hendges (2010), Redivo and Sornberger (2012) dentre outros.

\section{Resultados}

Por meio da análise dos processos de trabalhos correlatos e seguindo as normas sugeridas para o processo de rastreabilidade, foi criado um fluxo da rastreabilidade como ilustra a Fig. 1.

Com a constituição do fluxo do processo, foi iniciada o levantamento dos termos em cada etapa para a construção da ontologia. O fluxo criado demonstra as etapas que serão monitoradas no processo de rastreabilidade na cadeia produtiva de sementes.

Com base no alinhamento do fluxo, as produtoras de sementes devem realizar, para cada processo, o planejamento geral, os ajustes nos procedimentos e critérios estabelecidos, definir os documentos e critérios necessários, a fim de atender a estratégia da organização, requisitos dos clientes, aspectos de qualidade e requisitos legais.

Para a esquematização da ontologia de rastreabilidade na cadeia produtiva de sementes, foram consideradas sessenta e nove classes como, por exemplo, Armazenamento, Cultivo, Semente, Talhão, Adubação, Semeadura, Colheita, Campos, Insumos, Plantio, Equipamentos, Transporte, Beneficiamento, Fornecedores, Clientes, Cooperativas, Agrônomos, Produtoras, entre outras.

Algumas classes foram hierarquizadas em subclasses como, por exemplo, as classes Equipamentos e Semente por estas possuírem diversos tipos. A classe Equipamento tem as subclasses: Secador, Espirais, Padronizador, Moega, Tratadora, Mesa de Gravidade, Colhedoras e Balança Ensacadora; enquanto que Semente tem as subclasses: BetaSpp, Poaceae, BrasicaSpp e Fabaceae, referentes a famílias de plantas.

Para cada relacionamento existe a definição de um 


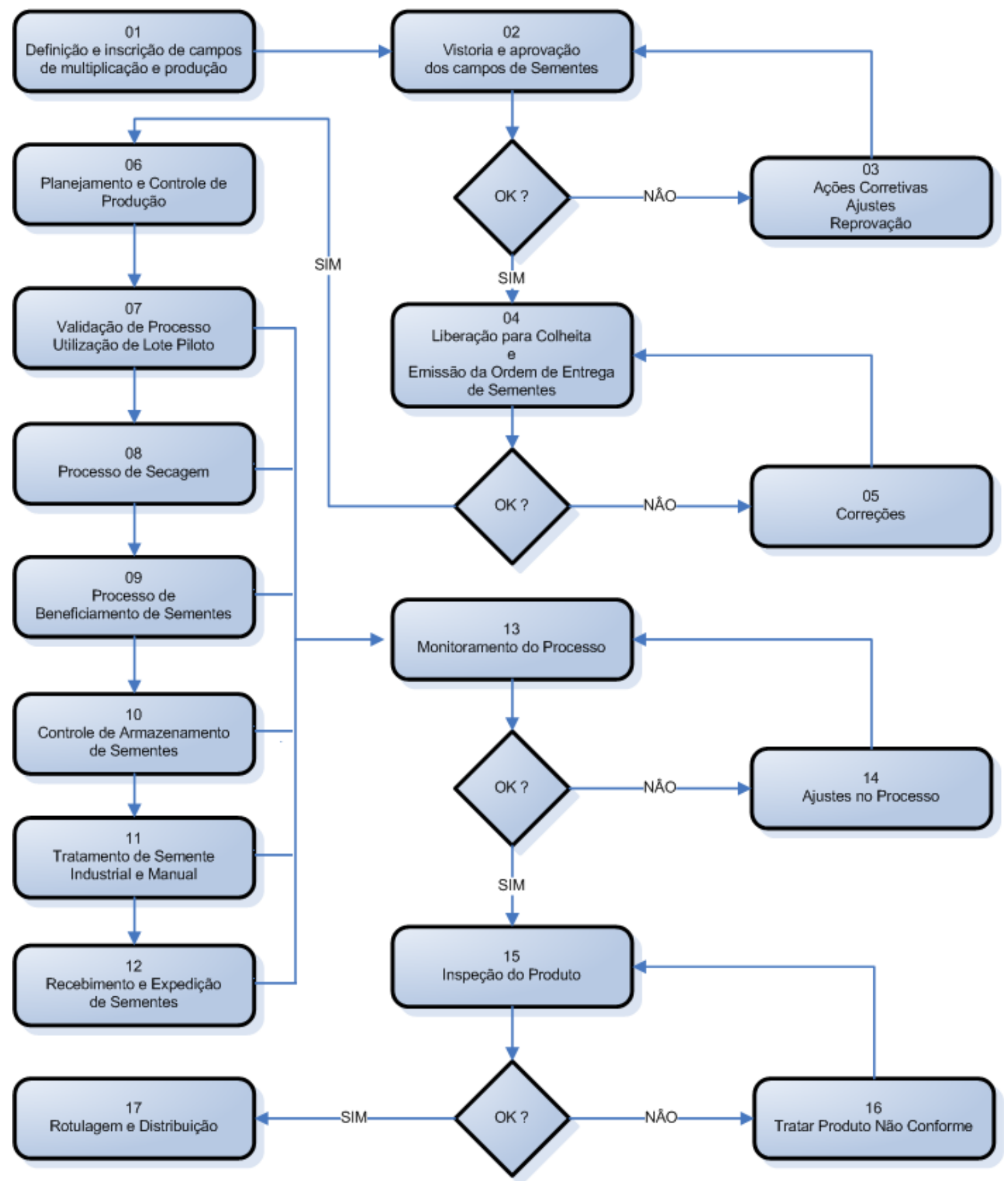

Figura 1: Fluxo do processo da rastreabilidade de sementes 

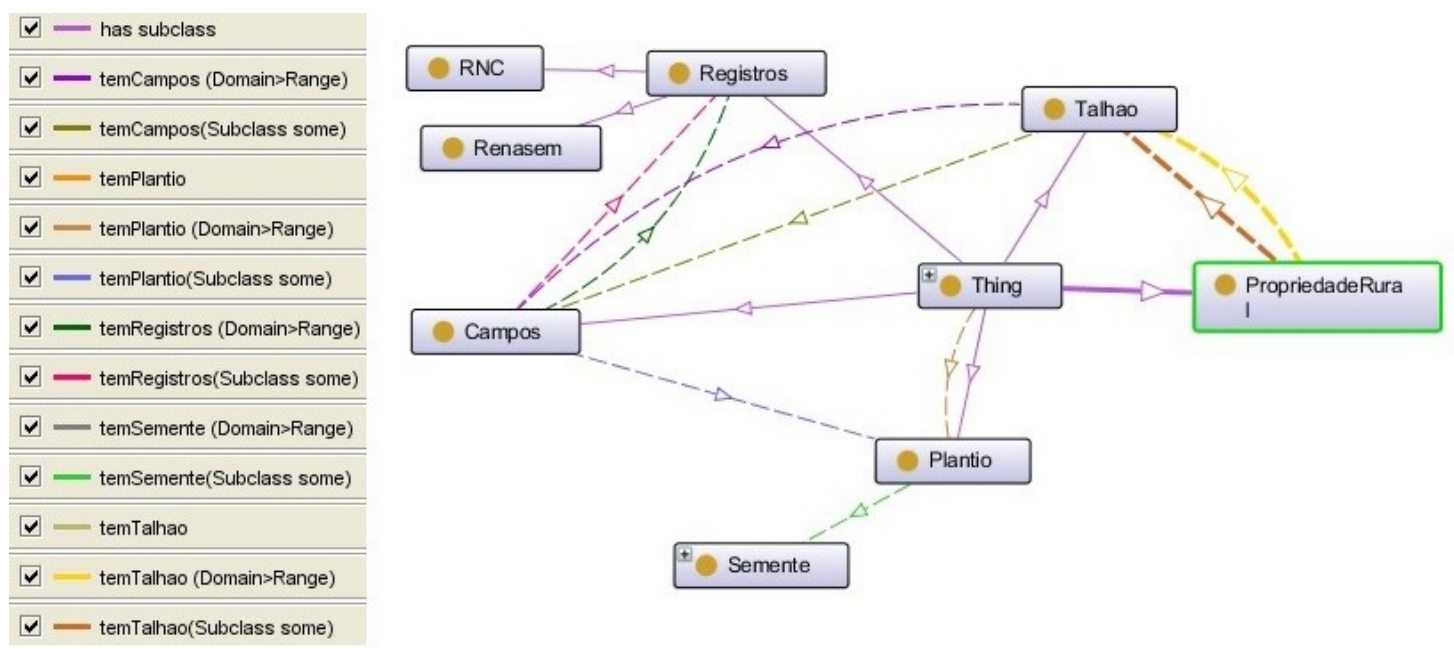

Figura 2: Relação entre classes

nome, com os nomes dos conceitos fonte e destino. A classe Campos, por exemplo, está relacionada com a classe Registros por meio da propriedade temRegistros, além disso, está relacionada com a classe Plantio com a propriedade temPlantio. Na Ferramenta Protégé(C) cria-se uma propriedade (neste caso do tipo Object Properties) e através desta propriedade são criadas as ligações necessárias entre as classes.

A propriedade ou relacionamento entre as classes é apresentada por meio do SubClassOf, que relaciona uma classe "filha" com uma classe "pai". A definição detalhada dos relacionamentos entre as classes gera o conjunto que descreve a semântica do domínio.

As relações existentes na ontologia são necessárias para estabelecer os diferentes relacionamentos entre as classes e subclasses. Como exemplo dessas relações na ontologia proposta, pode-se citar a relação temSemente, que relaciona as classes Semente e a classe Plantio do modelo. (Fig. 2). Na definição desta propriedade, somente o range que define as classes permitidas na propriedade, devido a relacionar-se com outras classes na ontologia.

Com a utilização de reasoners é possível realizar inferências sobre uma ontologia. Na Fig. 3, utilizando o Algoritmo Pellet, inicializa-se o Reasoner e depois com o uso da DL Query é demonstrada a inferência na Classe Semente.

Com o uso da DL Query podem ser visualizadas as subclasses e os indivíduos pertencentes à classe Semente. O Indivíduo Soja, por exemplo, pode ser inferido que é do tipo Fabacea, que é uma SubClasseOf da classe Semente.

Algumas das questões de competência que podem ser respondidas pela ontologia proposta são:

- Quais as propriedades rurais são controladas?

- Quais as propriedades rurais pertencentes um talhão?

- Quais as sementes presentes num talhão?

- Qual o código de registro de uma semente?

- Quais as características da semente Poaceae?

- Quais as características da semente Fabaceae?

- Quais os tipos de análises que as sementes foram submetidas?

- Que tipos de equipamentos são utilizados nas culturas?

- Que tipo de semente é a Soja?

- Que tipo de semente é o Trigo?

- Qual a data de plantio de um campo de produção?

- Qual a data da colheita de um campo de produção?

\section{Conclusão}

O objetivo principal deste artigo foi apresentar uma ontologia para rastreabilidade na cadeia produtiva de sementes, a fim de garantir a qualidade e a melhoria dos processos, por meio da representação do conhecimento. A ontologia descreve desde o início do processo de plantio, registros, colheita até o produto final envasado, ou seja, a ontologia pode oferecer uma visão ampla para o domínio.

A criação da ontologia reforça a utilização da definição do conhecimento do domínio como ferramenta de gestão de informações, qualidade e transparência em todas as etapas do processo produtivo. Essa é uma maneira de formalizar conhecimento de um determinado domínio, utilizá-la em larga escala e reutilizar em outras aplicações.

A principal contribuição da criação da ontologia, envolve a aplicação de modelos e ferramentas da Engenharia do Conhecimento, permitindo uma melhor representação dos elementos envolvidos no processo da cadeia produtiva de sementes.

Os resultados obtidos foram satisfatórios evidenciando o potencial diferencial das ontologias, pois proporcionam, a criação do conhecimento de um domínio que poderá ser estendida por vários pesquisadores e profissionais que tenham interesse em dar continuidade na pesquisa e aprimoramento da ontologia. Com a criação de regras lógicas, automatizam os processos, o compartilhamento e a reutilização do conhecimento modelado na ontologia para uso de profissionais na área.

Como trabalhos futuros, sugere-se efetuar aplicações de novos elementos e estender a ontologia. Também, se pretende buscar novos conhecimentos a partir do modelo criado, pesquisando as normas e legislações internacionais para a criação em padrão em língua inglesa. Ampliar o estudo sobre a utilização da ontologia para expandir o conhecimento 


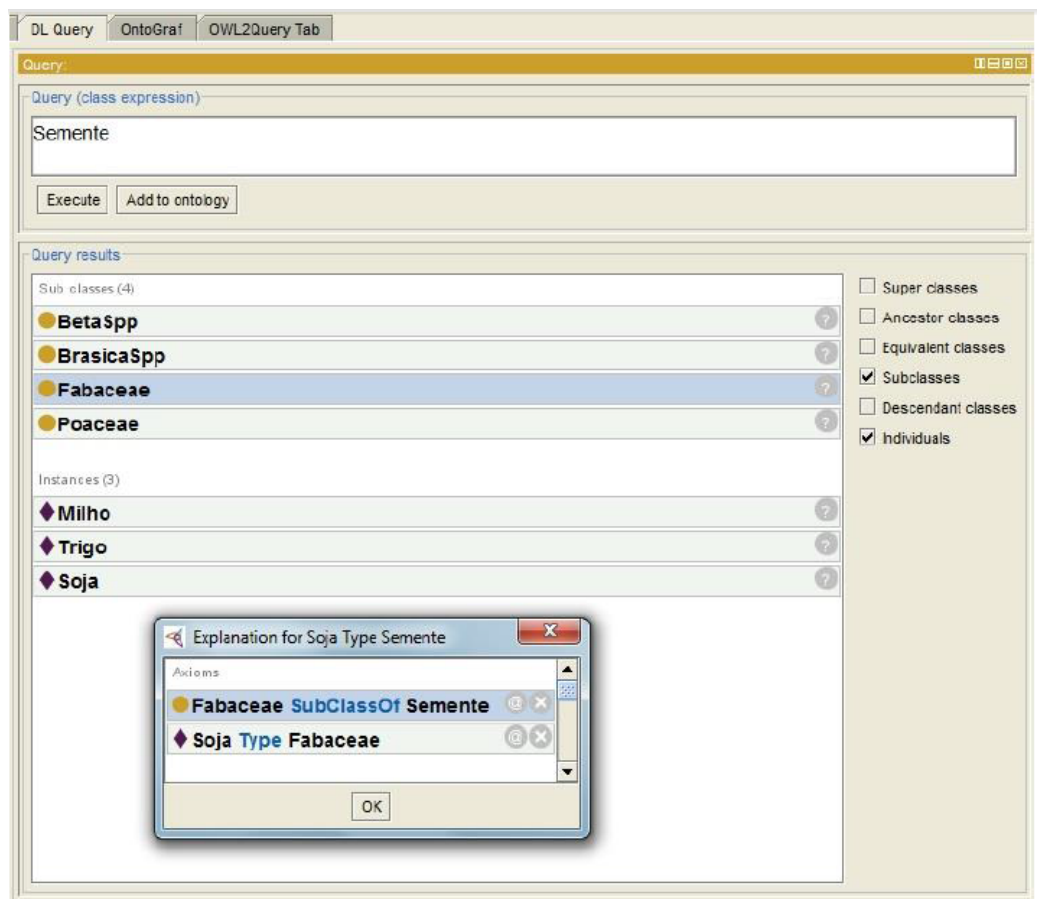

Figura 3: DL Query da classe Semente

sobre dados a serem integrados para realização de mineração de dados.

\section{Agradecimentos}

Os autores agradecem ao Programa de Pós-graduação em Computação Aplicada da Universidade Estadual de Ponta Grossa e a CAPES (Coordenação de Aperfeiçoamento de Pessoal de Nível Superior), pelo suporte financeiro para o desenvolvimento dessa pesquisa.

\section{Referências}

Abrahão, E. and Hirakawa, A. R. (2017). Task ontology modeling for technical knowledge representation in agriculture field operations domain, 2017 Second International Conference on Information Systems Engineering (ICISE), Charleston, SC, USA. https: //doi.org/10.1109/ICISE. 2017.18.

Andrade, K. d. (2008). A contribuição da engenharia de requisitos na especificação de ontologias: aplicação em redes colaborativas, Dissertação de mestrado, Universidade do Porto, Faculdade de Engenharia, Porto, Portugual.

Badia-Melis, R., Mishra, P. and Ruiz-García, L. (2015). Food traceability: New trends and recent advances. a review, Food Control 57: 393 - 401. https://doi.org/10.1016/j.foodcont.2015.05.005.

Beck, H., Morgan, K., Jung, Y., Grunwald, S., Kwon, H. and J., W. (2010). Ontology based simulation in agricultural systems modeling, Agricultural Systems 103(7): 463-477. https://doi.org/10.1016/j.agsy . 2010.04.004.

Blaudete, L. and Vilela, F. A. (2007). Unidades de beneficiamento de sementes, Revista SEED News 11(2).

Corcho, O., Fernández-López, M., Gómez-Pérez, A. and A., L.-C. (2005). Building legal ontologies with methontology and webode, in V. Benjamins, P. Casanovas, J. Breuker and G. A. (eds), Law and the Semantic Web. Lecture Notes in Computer Science, Springer Berlin Heidelberg, Berlin, Heidelberg, pp. 142-157. https://dx.doi.org/10. 1007/978-3-540-32253-5_9.

Dietz, J. (ed.) (2006). Enterprise Ontology, Springer, Berlin.

Fernandes, J. M. C. and Tibola, C. S. (eds) (2009). Manual técnico de rastreabilidade para cadeia produtiva do trigo, Embrapa Trigo, Passo Fundo.

Gazzola-Neto, A., Aumonde, T. Z., Pedó, T., Levinski, P., Fonseca, F. d. R., Peske, S. T. and Villela, F. A. (2012). Rastreabilidade aplicada à produção de sementes de soja, Informativo Abrates 22(2): 20 24.

GS1 (2012). Standards Document Business Process and System Requirements for Full Chain Traceability. Available at https://www.gs1.org/docs/ traceability/Global_Traceability_Standard.pdf (Accessed 6 nov 2018).

Guariano, N. (1998). Formal ontology and information systems, Proceedings of FOIS'98, FOIS' 98, Trento, Italy.

HACCP (2009). Principles e Application Guidelines, 3 edn, FDA's CFSAN Laboratory Quality Assurance Manual College Park, Maryland.

ISO:22005 (2007). Traceability in the feed and food chain - General principles and basic requirements for system design and implementation. Available at https:// 
www.iso.org/standard/36297.html (Accessed 6 nov 2018).

Kwon, H., Grunwald, S., Beck, H. W., Jung, Y., Daroub, S. H., Lang, T. A. and Morgan, K. T. (2010). Ontology-based simulation of water flow in organic soils applied to florida sugarcane, Agricultural Water Management 97(1): 112 - 122. https://doi.org/10. 1016/j. agwat. 2009.08.019.

Leonelli, F. C. V. (2006). Rastreabilidade integrada de grãos (rig): um modelo de referência, Anais do Encontro de Engenharia De Produção, Fortaleza.

Macedo, S. X. and Dias, M. M. (2016). An ontology of diseases caused by improper use of pesticides, Revista Electronica de Sistemas de Informaçao 15(1): 114. https://doi.org/10.21529/RESI . 2016.1501003.

Melo, D. P. d. (2017). Ontologia de ciclo de exploração agrícola, Dissertação de mestrado, Universidade Católica de Brasília, Brasília, Brasil.

Mohan, K. and Ramesh, B. (2002). Managing variability with traceability in product and service families, Proceedings of the 35th Annual Hawaii International Conference on System Sciences (HICSS'02), IEEE Computer Society, Big Island, HI, USA, pp. 1309-1317. https://doi.org/10.1109/HICSS . 2002.993995.

Musen, M. (2015). The protégé project, AI Matters 1(4): 4-12. https://doi.org/10.1145/ 2757001.2757003.

Noy, N. F. and Mcguinness, D. L. (2001). Ontology development 101: A guide to creating your first ontology, Technical report, Stanford University.

Papajorgji, P. J. and Pardalos, P. (eds) (2009). Advances in Modeling Agricultural Systems, Springer, Boston, MA.

Redivo, A. and Sornberger, G. P. (2012). Rastreabilidade na produção de semente de arroz: um estudo de caso, XXXII Encontro Nacional de Engenharia de Produção, ENEGEP, Bento Gonçalves, RS, Brasil. Available at http://www .abepro.org.br/ biblioteca/enegep2012_TN_STP_158_922_20089.pdf (Accessed 8 Out 2018).

Semeráth, O., Barta, A., Horváth, A., Szatmári, Z. and Varró, D. (2017). Formal validation of domainspecific languages with derived features and well-formedness constraints, Software \& Systems Modeling 16(2): 357-392. https://doi.org/10.1007/ s10270-015-0485-x.

Silva, J. d. (2018). Ontologia na rastreabilidade de dados agrícolas, Dissertação de mestrado, Universidade Estadual de Ponta Grossa, Ponta Grossa, Brasil.

Sure, Y., Staab, S. and Studer, R. (2004). On-toknowledge methodology (otkm), in S. Staab and R. Studer (eds), Handbook on Ontologies, Springer Berlin Heidelberg, Berlin, Heidelberg, pp. 117-132. https://doi .org/10.1007/978-3-540-24750-0_6.

Tillmann, C. A. d. C. (2006). Modelo de sistema integrado de gestão da qualidade para implantação nas unidades de beneficiamento de sementes, Tese de doutorado, Universidade Federal de Pelotas,
Faculdade de Agronomia Eliseu Maciel, Pelotas, Brasil.

W3C (2018). World wide web consortium. Available at https://www.w3.org/ (Accessed 8 Out 2018).

Zanatta, T. d. C. and Hendges, T. (2010). Implantação da rastreabilidade na multiplicação de sementes de soja, Revista Científica da Faculdade de Balsas 1(1). 